\title{
MAKER: Applications of 3-D Printing and Laser Cutting in the Development of Autonomous Robotics
}

\section{William F Cohen, OSU Department of Engineering Education}

I am a graduate teaching assistant for the Fundamentals of Engineering for Honors program at the Ohio State University, which is a 2 semester course sequence for first-year engineering students focusing on programming in $\mathrm{C} / \mathrm{C}++$ and MATLAB, computer aided drawing in SolidWorks, and a 10 week autonomous robot design project.

\section{Jacob J. Enders, The Ohio State University}

Jacob J. Enders is a Biomedical Engineering honors student at The Ohio State University and an Undergraduate Teaching Assistant for the Fundamentals of Engineering for Honors program. He is a member of the Eminence Fellows scholarship program and is a research assistant studying cancer cell migration and metastasis using DNA force sensors. He will graduate with his B.S.B.M.E. in May 2018.

\section{Kelly L. Kolotka, The Ohio State University}

B.S. Chemical Engineering, Minor: Biomedical Engineering, Class of 2018 Undergraduate Teaching Assistant for the FEH program Department of Engineering Education

\section{Richard J. Freuler, The Ohio State University}

Richard J. Freuler is the Director for the Fundamentals of Engineering for Honors (FEH) Program in the OSU Department of Engineering Education. He teaches the two-semester FEH engineering course sequence and is active in engineering education research. He is also a Professor of Practice in the Mechanical and Aerospace Engineering Department and conducts scale model investigations of gas turbine installations for jet engine test cells and for marine and industrial applications of gas turbines at the Aerospace Research Center at Ohio State. Dr. Freuler earned his Bachelor of Aeronautical and Astronautical Engineering (1974), his B.S. in Computer and Information Science (1974), his M.S. in Aeronautical Engineering (1974), and his Ph.D. in Aeronautical and Astronautical Engineering (1991) all from The Ohio State University.

\section{Dr. Deborah M. Grzybowski, The Ohio State University}

Dr. Deborah Grzybowski is a Professor of Practice in the Department of Engineering Education and the Department of Chemical and Biomolecular Engineering at The Ohio State University. She received her Ph.D. in Biomedical Engineering and her B.S. and M.S. in Chemical Engineering from The Ohio State University. Her research focuses on making engineering accessible to all students, including students with visual impairments, through the use of art-infused curriculum and models. Prior to becoming focused on student success and retention, her research interests included regulation of intracranial pressure and transport across the blood-brain barrier in addition to various ocular-cellular responses to fluid forces and the resulting implications in ocular pathologies. 


\section{MAKER: Applications of 3D Printing and Laser Cutting \\ In Development of Autonomous Robotics}

\section{Introduction}

During the final semester of the Fundamentals of Engineering for Honors (FEH) program at The Ohio State University, first-year engineering students are tasked with a ten week autonomous robot design project. The project is intended to teach the basic principles of the engineering design process, mechanical and electrical design, programming, Computer Aided Design (CAD), project documentation, management, and teamwork. Interdisciplinary teams of four students must design, build, and program a completely autonomous robot that is capable of traversing a well-defined course and completing a series of objective tasks. Possible objectives include avoiding obstacles, pushing buttons, flipping switches, distinguishing colored LED lights, and lifting, transporting, and depositing objects. Students are concurrently introduced to Computer Aided Design software, SolidWorks (Dassault Systèmes SolidWorks Corp., Paris, France) since 2012, throughout the project to aid in the design process.

Students are supplied with a microcontroller to both power and control their robot. The controller includes a multitude of DC motor, servo motor, and digital and analog I/O ports, which can be used to control the drivetrain, sensors, and mechanisms on the robot. All necessary robot parts for student use including motors, sensors, hardware, wiring supplies, chassis materials, and other miscellaneous items are supplied by FEH through a mock store website where students can make purchases.

3D printing and laser cutting technology is made available to students to enhance the design process. These technologies allow students to create custom designed mechanisms and chassis in order to complete the given objectives. Desired parts can be submitted through the FEH Store website to be fabricated by the instructional staff. Since introducing these technologies into the project, the complexity and intricacy of students' designs has increased and proven to be very effective in such an open-ended design project.

\section{Autonomous Robot Project Description}

Each year, a new robot course is developed by the student instructional staff (undergraduate and graduate teaching assistants) to provide each class of first-year engineer students with a unique, cutting-edge experience ${ }^{1}$. Students are provided with a Scenario Document prior to starting the design project, which outlines the project in great detail, including information about the course specific objectives, rules and constraints, stock parts available for purchase, important dates and deadlines, and much more. The remainder of this paper will focus on the project scenario from spring 2015, Artic Exploration.

The following excerpt describes the Artic Exploration scenario as it was given to the students. The bracketed numbers correspond to Figure 1 and Figure 2, shown below: 
"A group of workers for an engineering and research collaborative in a desolate Arctic area are updating an oil infrastructure project to meet increased demands. A Base of Operations (BOO) [1] has been established in the area of interest and work has commenced. Extreme weather is common and frequently disables the main power system, making working conditions unacceptable. Due to the importance of the project and existing infrastructure, the project team desires an autonomous robot that can accomplish a variety of repair, maintenance, and risk-mitigating tasks at the project site that are unsafe for humans to attempt.

A research team at The Ohio State University has constructed a scale model of the BOO and the project site on which each robot prototype will be tested. The robot will be evaluated on its overall design and performance on the course and be expected to complete its tasks in a timely and safe manner.

The course is designed to accommodate up to four robots operating simultaneously, each competing against the others to successfully complete specified tasks in the most efficient manner. Each robot will begin in its respective Starting Area [2]. At the start of the simulation, a red LED in the center of the Starting Area will illuminate to signal the robot to begin operations. It will then move about the course to complete the given tasks. Each Robot is responsible for maintaining its individual portion of the course. The Fences [3] around the Power Poles and behind the Crank separate each robot's respective section of the course. Intentional robot-robot interaction and breaching of boundaries is prohibited.

The robot must detect the start signal and complete several independent tasks. The tasks may be completed in any order, except for resetting the Oil Pump, which must be completed last. The robot must bring the Salt Bag [4] from the supply zone at the bottom of the project site to the Garage [5] in the BOO. A wireless communication system, the Robot Positioning System (RPS), will transmit a signal to the robot containing information on temperature, soil conditions, and other data from various remote locations around the project site. To inform the workers inside the BOO, the robot must press the 3 buttons on the wall of the BOO [6] in a specified order based on the signal from the RPS. Due to severe weather, one of the two main Power Poles [7] has been knocked down. To restore main power, the robot must return the downed power pole to its original upright position by turning the Crank [8] until the pole is upright. A LED Light [9] on the course will indicate the direction the robot should turn the crank for total restoration of operational conditions. For the last task, the robot must approach the Oil Pump [10] and trigger the manual shutdown switch in the correct direction. The correct direction to flip the switch will be sent through a wireless RPS signal. Once this task is completed, timing on the course will stop and the run will be complete.

Multiple obstacles exist on the top level of the project site, including three Trees [11] and a Snow Pile [12] on the road near the BOO. Neither the snow pile nor trees are fixed in place. The robot may move the snow pile at its discretion but should avoid knocking over or moving the trees.” 


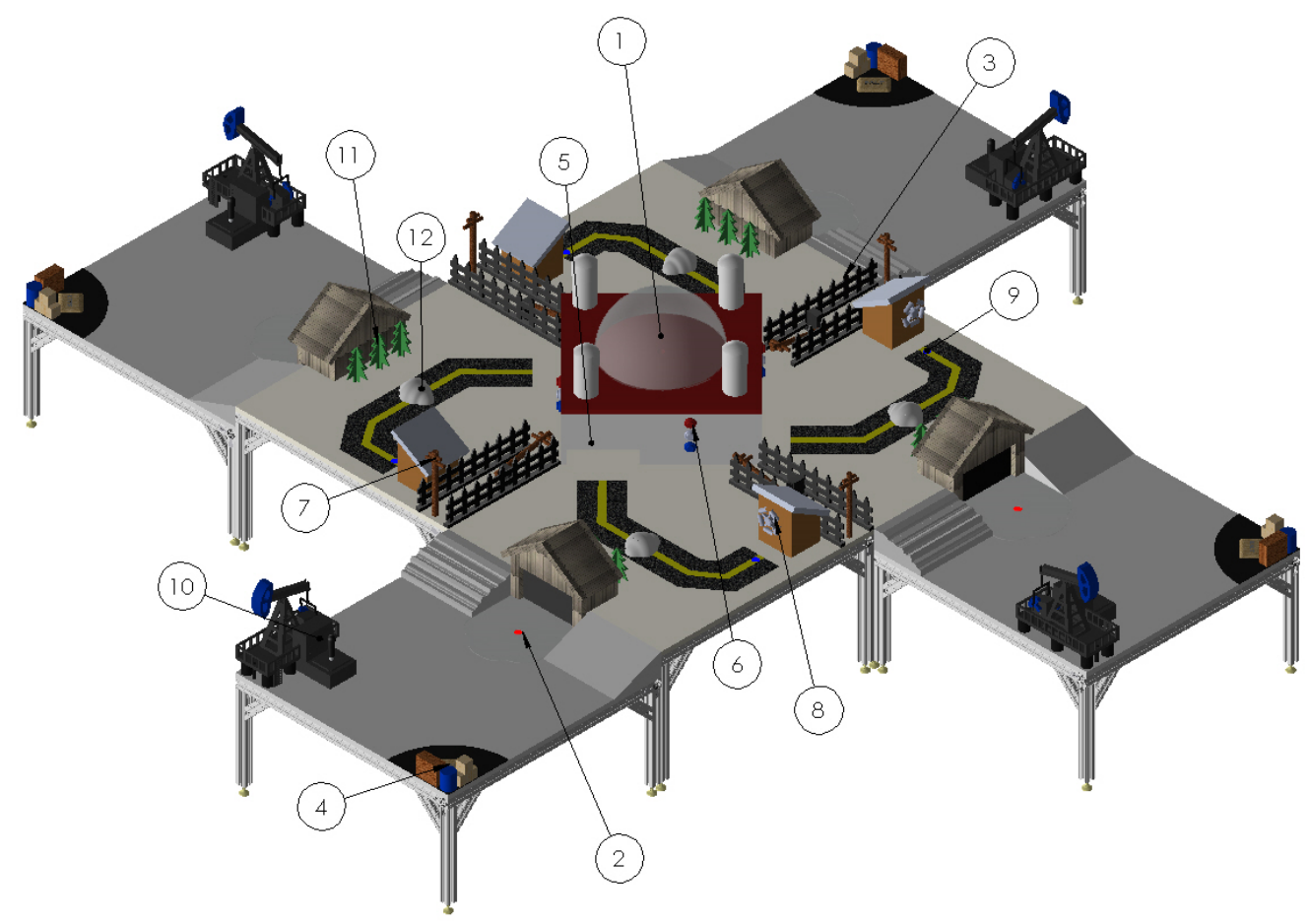

Figure 1: SolidWorks model of 2015 FEH Robot Course: Arctic Exploration

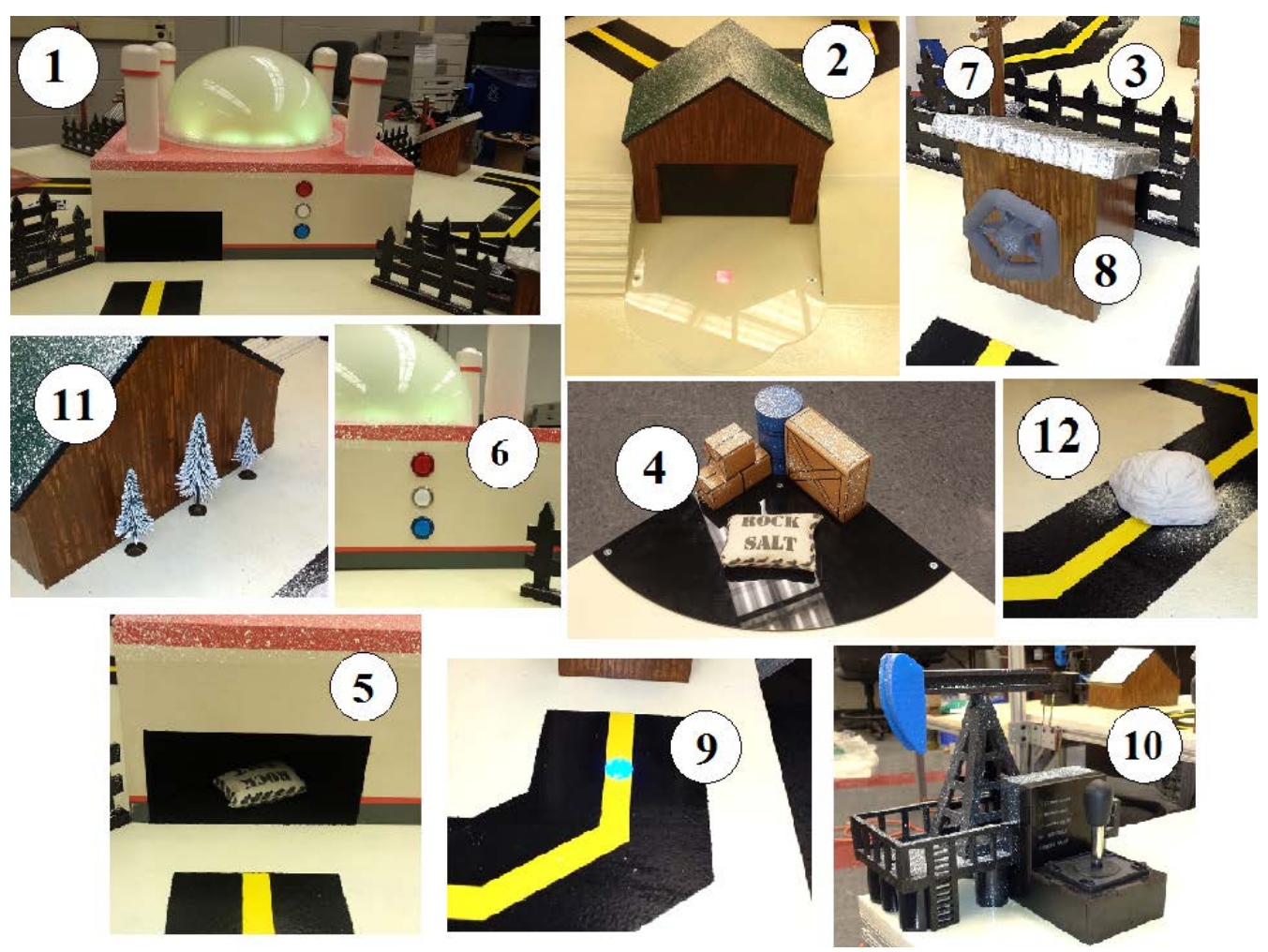

Figure 2: Photographs of the 2015 FEH Robot Course: Arctic Exploration 
Each course section is approximately a $3 \times 6$ foot rectangle, with 4 course sections arranged in a windmill pattern to create a full course. A robot is only responsible for completing the tasks in a single course section during a run. Figure 3 below shows a top view of a single course section.

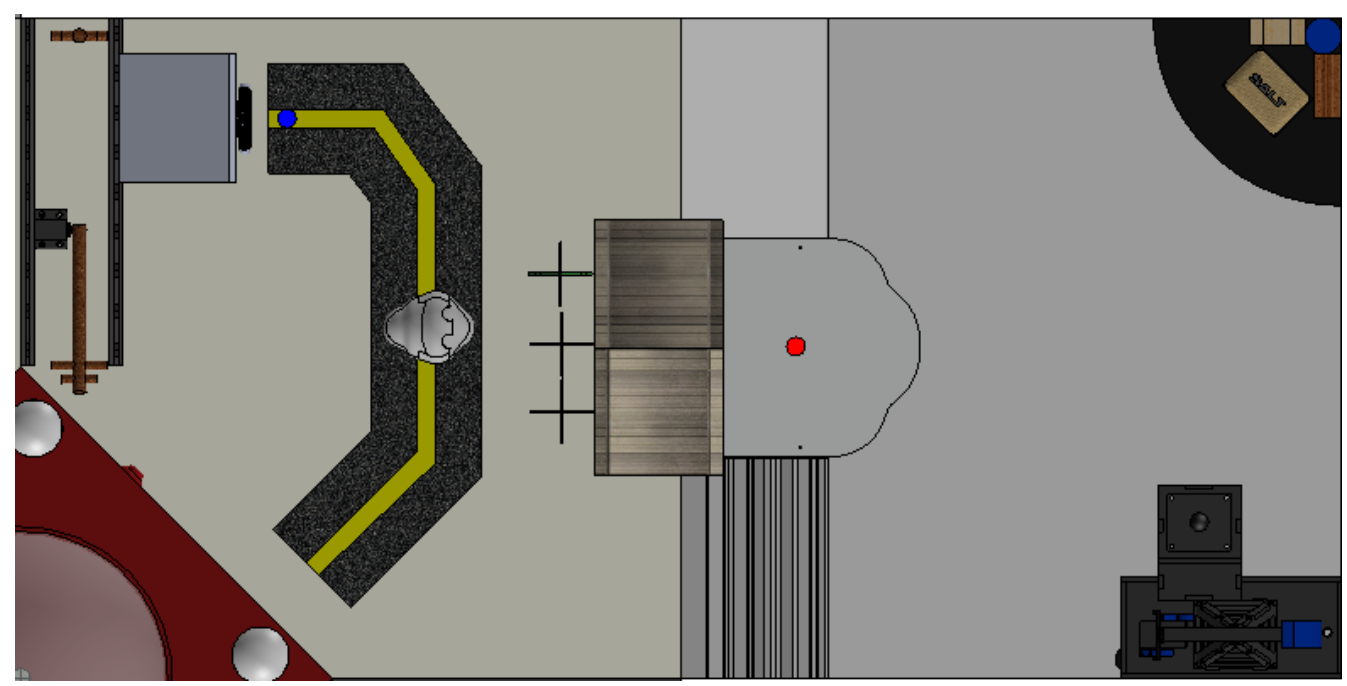

Figure 3: Top view of a single course section of the 2015 FEH Robot Course: Arctic Exploration

Additional project constraints include:

- Robots must fit within a footprint no larger than 9x9 inches and have a maximum height limit of 12 inches.

- Robots must be completely autonomous with no outside control.

- Fabrication of the robot must be solely by members of the team. No outside help is allowed.

In order to complete the tasks specified in the given scenario, teams are loaned a programmable microcontroller in order to provide power and control their robot ${ }^{2}$. Additionally, teams are given a budget limit of $\$ 160$ to purchase necessary materials including DC motors, servo motors, sensors, pre-fabricated robotic parts, axles, hardware, lumber, sheet metal, and other miscellaneous items. All materials are supplied directly through the FEH program by making purchases through a mock website, the FEH Store. Teams also have the option of designing their own 3D printed or laser cut parts, which can be purchased through the Store website and manufactured by the instructional staff.

\section{D Printing}

Students are given the option of using 3D printing to design custom mechanisms or other unique parts to aid in the design process. Many students chose to use 3D printing for uniquely shaped parts that make a specific course challenge easier or serve as multipurpose mechanisms. Once a 
student has designed and submitted a design to be printed, the part is printed by designated undergraduate teaching assistants using a Makerbot Replicator 2 and PLA filament. Due to the flexibility of designing completely custom parts and the complexity of parts that can be fabricated, a wide variety of very different robots and mechanisms are created that are all capable of completing the course objectives in a multitude of distinctive and fascinating ways.

\section{Part Design}

Students are instructed in how to use the CAD software SolidWorks during the first three weeks of the second semester of the Fundamentals of Engineering for Honors program. Following this, the students are provided with documentation on the goals of the project and the various tasks the robot must complete. Students then go through a rigorous brainstorming process in which they can identify places on the course where 3D printed parts may be useful. Once the instructional staff approves the teams' robot designs, they are able to submit 3D part designs for printing.

The precision of 3D printed parts is based on the layer thickness used in the slicer software. For the Makerbot Replicator 2 used for this project, the layer thickness is set to $0.1 \mathrm{~mm}$. Therefore no dimensions should be designed more precisely than with a $\pm 0.1 \mathrm{~mm}$ tolerance. Additionally, 3D printed parts have the potential to break easily if not designed properly. Students are instructed to make features thicker than 0.05 ” to decrease the chance of parts fracturing. In addition, where features meet at 90 degree angles, students must add fillets to their designs. An example of the addition of fillets is shown below in Figure 4. Where the cylindrical prongs meet the circular base, the fillets increase the joint surface area, which in turn increases the strength of the joint.

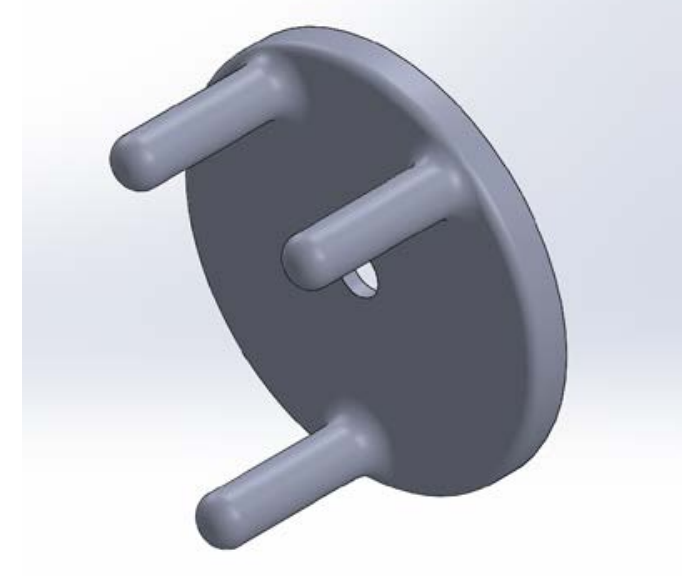

Figure 4: Fillets on cylinders joined to circular base of 3D print model.

Designs should also minimize base area to prevent warping. As the PLA filament cools after extrusion, it may contract on the build plate, and can potentially come off the plate and warp the part. Minimizing contact surface area lowers the amount of the material that can contract to cause warping. In addition to base area, complex structures with large overhangs tend to not print as well because supports are required to prevent sagging of the material. Supports can be difficult to remove from printed parts and may make the surface rough and uneven. Such 
supports then may make it difficult to join the part to other structures with specific tolerance requirements.

CAD files created in SolidWorks for 3D printed parts must be exported as an .stl file and submitted through the FEH Robot Store website to be fabricated by the instructional staff. Depending on the size, parts can take between 1 and 4 hours to complete, and printed parts are delivered and made available in the Store to students within 2 days. A limit of 100 grams of PLA filament is set to deter students from solely relying on 3D printing and to consider other possible construction options when applicable. Students are charged $\$ 0.25$ per gram of filament used, which is deducted from their $\$ 160$ budgets upon receiving their parts from the store.

\section{Multipurpose and Lightweight Parts}

Through using 3D printing, students are able to design and fabricate custom parts for use on their robots to allow functionality that otherwise might be difficult to achieve. In past robot design projects, students have chosen to use 3D printing for uniquely shaped parts that make a specific course challenge easier or that can serve as multipurpose mechanisms.

An example of a multipurpose 3D printed mechanism created by a team can be seen below in Figure 5, Figure 6, and Figure 7. The mechanism produced was capable of pressing all three buttons on the BOO, turn the Crank, and flip the final switch on the Oil Pump. The two custom parts have a series of prongs in a desired geometry to aid in completing each task. The parts attach onto two servo motors allowing for two axis of rotation of the mechanism. Figure 7 shows the completely assembled robot with the attached mechanism on the front end.
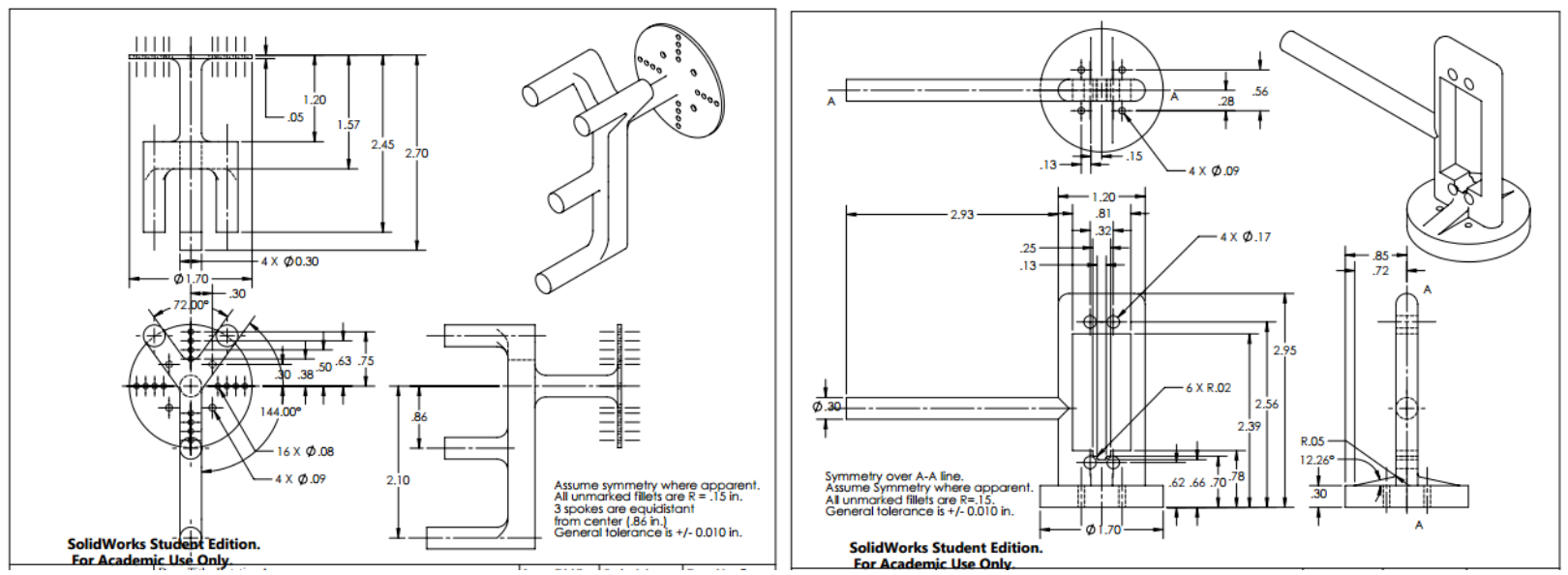

Figure 5: SolidWorks Dimensioned drawing file of Student Designed 3D printed servo motor attachments 


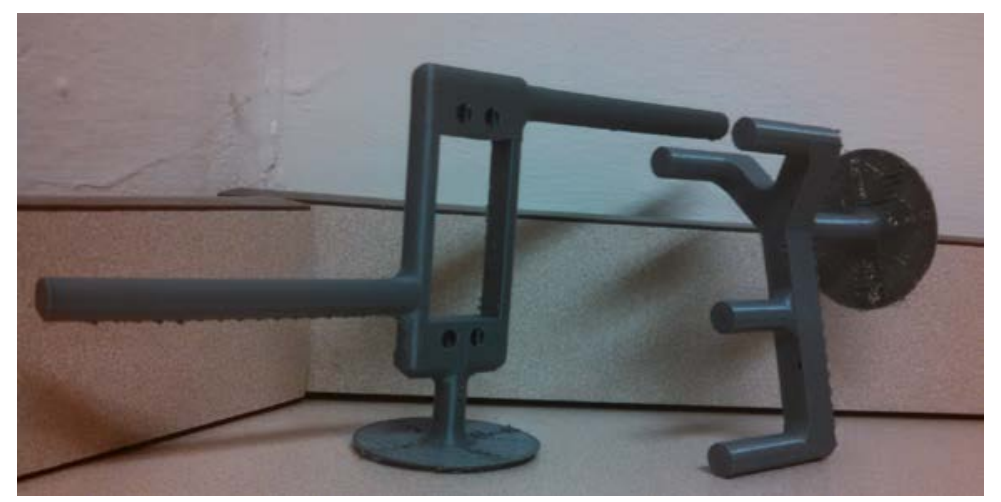

Figure 6: 3D printed servo motor attachments for pushing the buttons on the BOO and turning the crank

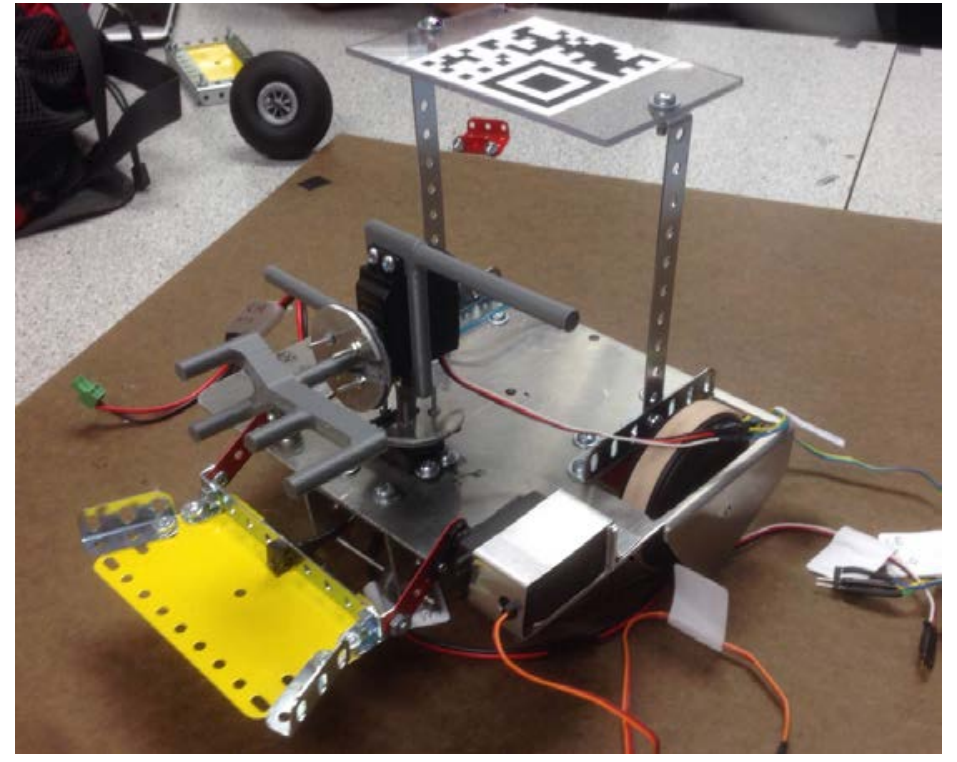

Figure 7: Fully assembled robot with custom 3D printed servo attachments

Students have also found 3D printing to be advantageous because parts are light weight when using PLA filament. Another team's robot employing 3D printing can be seen in Figure 8 . The team designed a large scooping mechanism, with a 3D printed part that attached directly to a servo motor. The scoop is an ideal size and shape to wedge under the Salt Bag and to lift, carry, and deposit the Salt Bag into the BOO. The scoop also has a small square hole near the back side to fit a microswitch inside. The microswitch was used as a sensor to determine if the robot had successfully picked up and lifted the Salt Bag. Such a design, if made of sheet metal or Erector set pieces, screws, and nuts, would be much heavier and could be difficult for a servo motor to rotate to pick up the salt bag. Thus, 3D printing allowed this team to make a large scoop easily able to perform the required tasks in the project. 


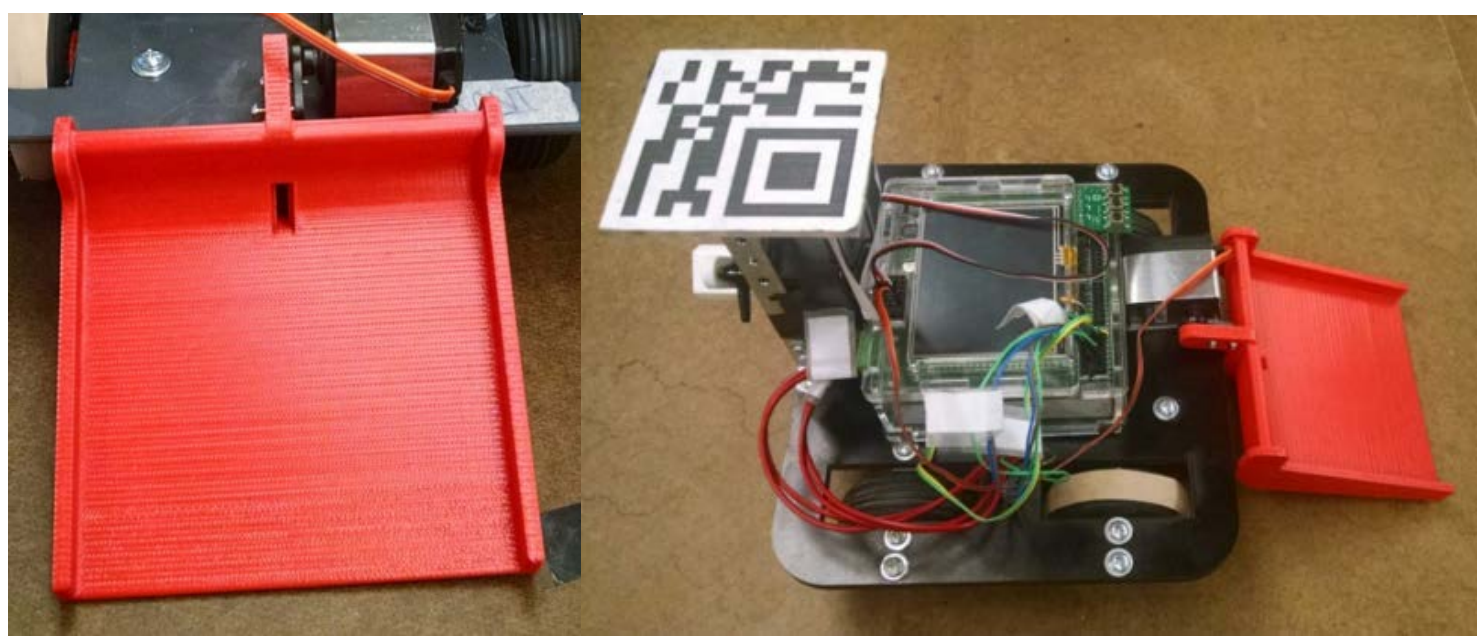

Figure 8: Robot with 3D printed scoop mechanism used to pick up and deposit the Salt Bag

\section{Design Sharing}

The FEH Store also provides pre-designed 3D printed parts including motor mounts and wheel adapters to make the assembly process as easy as possible. Pre-designed motor mounts are made to be a simple snap-fit to hold the motor in place, and the wheel adapter attaches directly into the spokes of the wheel to help fasten the wheel to an axle. The described parts are made available to all students because only specific motors and wheels are supplied by the FEH Store, and are therefore frequently needed by most teams.

Allowing teams to purchase these parts without having to spend time in the design phase gives them greater opportunity to work on more complex components for the project. However, students are given the CAD files for these parts and may choose to edit and resubmit a more customized part if they would like to make modifications to the design. The two examples of pre-designed parts described above can be seen in Figure 9 and Figure 10.
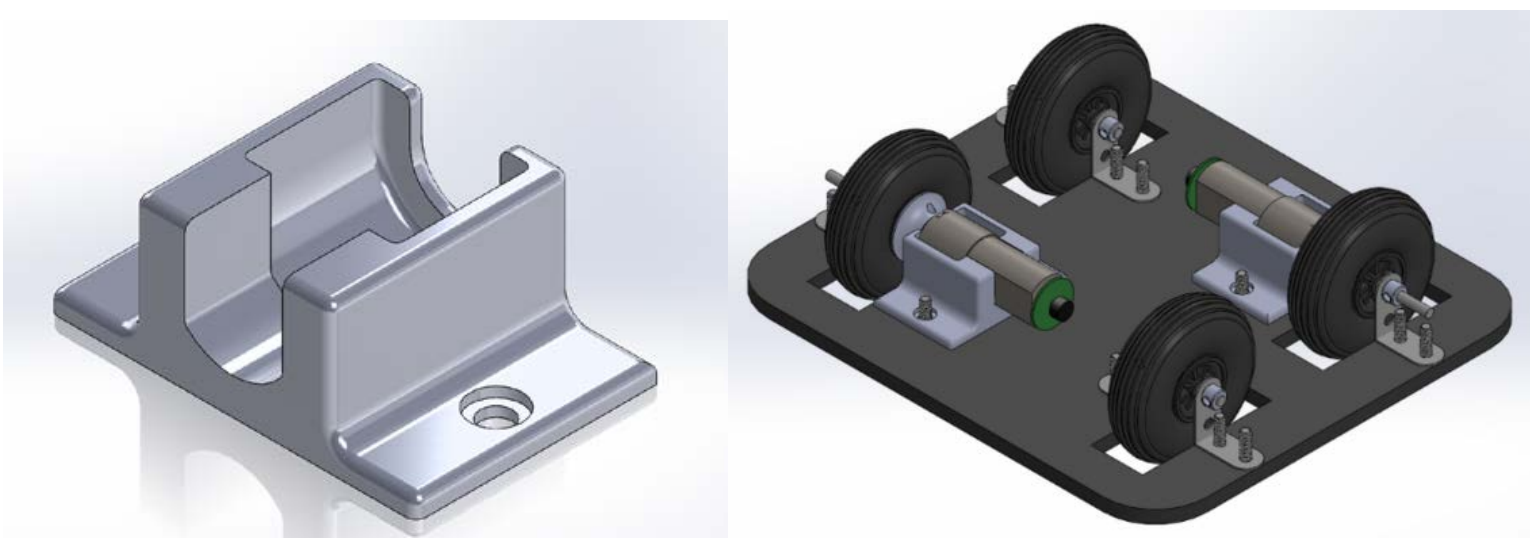

Figure 9: Pre-designed 3D printed snap-fit motor mount for student use 

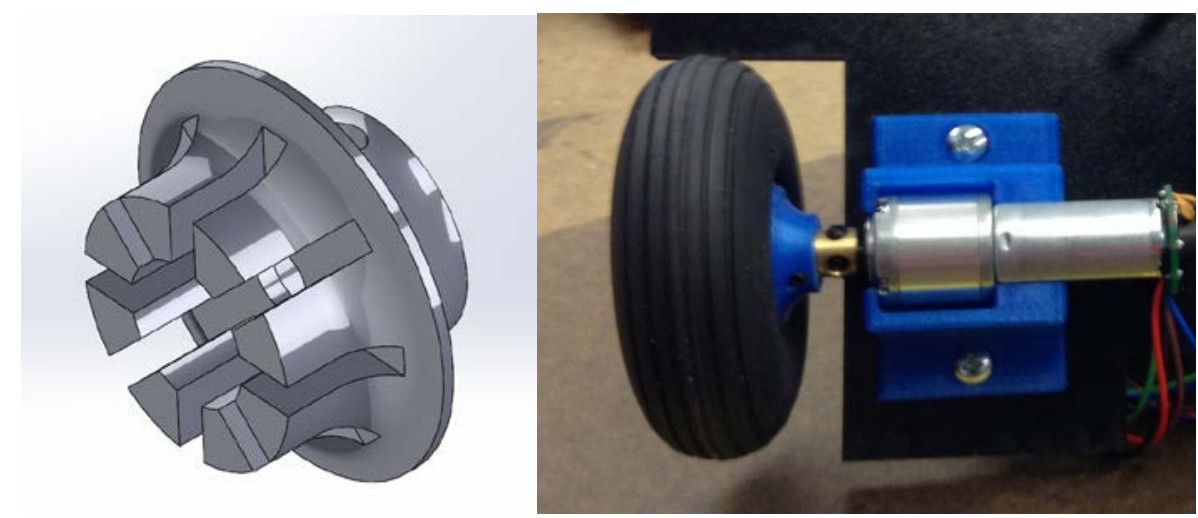

Figure 10: Pre-designed 3D printed snap-fit wheel to axle mount for student use

\section{Laser Cutting}

Students are also given the opportunity to use laser cutting as a means of construction. Laser cutting can produce extremely accurate cuts in a 2D plane, which can be useful for students who do not have much experience with machining and power tools. The laser can also be beneficial in ensuring that the details essential to the function of the part are accurate. This is particularly important when dealing with the drivetrain of the robot or other robotic parts that require specific tolerances. All laser cutting for this project is done using the Universal Laser Systems PLS6.75 laser cutter. Students choose to use this method because of the accuracy at which cuts can be made which allow for motors, sensors, and other mechanisms to be precisely located on the robot. Some teams also use laser cutting for other applications like complex arm mechanisms or wire management in order to complete the course in a unique and efficient manner.

\section{Part/Assembly Design}

Similar to 3D printing, students apply their coursework experience from earlier in the semester to design their own parts in SolidWorks. Laser cutting, too, requires that the instructional staff has approved the initial order and brainstormed ideas of the team. Specifically for laser cutting, the students are provided with documentation that outlines the specifics of the laser cutting process as well as tutorials for modeling with the CAD software. This includes specific tolerance values, cost per unit area of material used, cost based on laser path for each of the available materials, and a step-by-step guide to modeling successful parts.

The main advantage with laser cut parts is that they are very accurate when designed properly. When designing laser cut parts, the students are advised to take into account the kerf of the laser. On either side of the laser path, extra material is removed as it is cut. Therefore, when designing parts that require critical tolerances, the students must counteract this effect by offsetting their designs 0.003 ” away from the original cutting line. This value is specific to the laser used in the FEH program. During the design process, the team must also evaluate the average thickness and standard deviation for each type of material which will be detailed in a later section. 
For more complex assemblies rather than individual parts, the students are advised to modify drawings to include interlocking pieces and to consider how all of the parts will be fastened together. Two different strategies are provided for them in tutorials as they complete this process. The first suggestion has students first create individual pieces, and then create an assembly using SolidWorks to ensure that the parts intersect where desired. However, this strategy may require redrafting multiple parts. Another strategy recently devised by an undergraduate teaching assistant is to create the final assembly as one part by sketching on different planes as shown to the students earlier in the curriculum. Then, they create a sketch on each plane of their drawing to make individual flat parts. These are saved separately, and that area is removed from the original. The process is continued until one flat part remains. These files should still be verified by creating an assembly, but all parts should fasten together as the original did. This system is useful in both chassis design, as many teams include fenders for wheel protection, and for use in mechanisms such as arms with multiple joints.

After the designs are completed, students create a 2D vector graphic file for each laser cut part by selecting the principle face of their CAD part. Teams then submit their part files to the FEH Robot Store in order for the instructional staff to complete their order. In addition to the file, they must submit information about the length of the laser path and would face a recalculation fee if the values are not deemed accurate by the instructional staff. Each order also includes a \$2.00 setup fee, and a cost for the laser path, in addition to the surface area of the material used. Most parts take only a few minutes to be cut, but as with 3D printing, students are guaranteed to receive their completed order within 2 days.

\section{Material Selection}

Material options for laser cutting include 1/4” acrylic, 1/8” acrylic, and 1/4" laminated wood. Each material has advantages and disadvantages, which students must take into account when making a selection. The laminated wood is a lighter weight material and has the ability to be machined at any time should modifications become necessary. However, the laminated wood is more prone to wear from repeated use and can warp over time. The acrylic is a more structurally stable choice, but is prone to shattering when machined. Therefore, once an acrylic piece has been laser cut, it is extremely difficult to modify the part. Additionally, either thickness of the acrylic is more expensive per unit area than the laminated wood. Students are not given a limit on material for laser cutting, other than what they can afford in their \$160 budget.

The material to be used should be taken into account when the students are modeling their parts. Although is it recommended that they include any screw holes, wire management designs, or other special features to be included despite the material, it is particularly important for designs that are cut from the acrylic. As previously mentioned, it is not advised to manually modify parts cut from acrylic as they are likely to shatter. It is also pertinent during the design process to assess the kerf, average thickness, and standard deviation of each material as they are all different. This would show effects primarily with any interlocking tabs. The laminated wood requires less accurate tolerances for the kerf which is beneficial in more complex designs. When using the laminated wood, the chassis and mechanisms are lighter requiring less torque from the motors which allow designs to be sustainable over time. 


\section{Chassis and Drivetrain Design}

Two examples of robots with laser cut chassis can be seen below in Figure 11, Figure 12, and Figure 13. Both chassis use laminated wood, however each are distinct from one another. The first robot shown in Figure 11 and Figure 12 has a chassis made of five separate pieces. One of the five pieces is the base structure, where most of the components of the robot are attached. The other four pieces are fenders which protect the wheels and drivetrain underneath the robot. Figure 13 shows a second robot shown that employs a laser cut chassis made of one single base part. All other components of the robot are fixed to this base. Additionally, this chassis has large square holes near the left and right edges and in the center, allowing the wheels and gears of the drivetrain to sit higher up into the chassis and lowering the center of gravity of the robot. These are common designs for laser cut chassis as one prevents wear on the drivetrain while the other is a cost efficient method for a user friendly chassis.
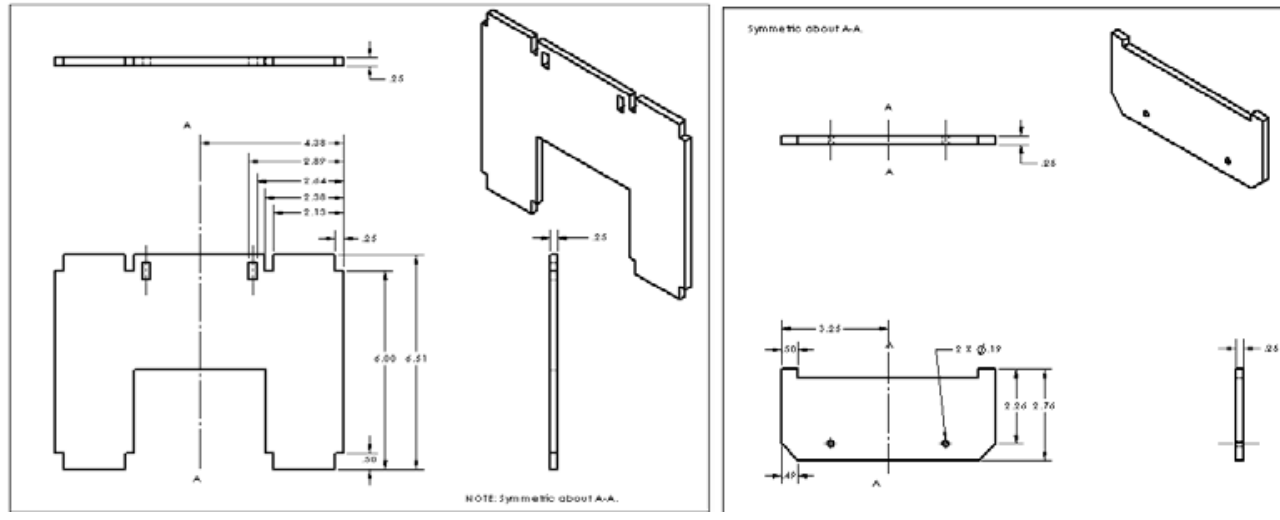

Figure 11: Dimensioned SolidWorks Drawing of Laser Cut Chassis

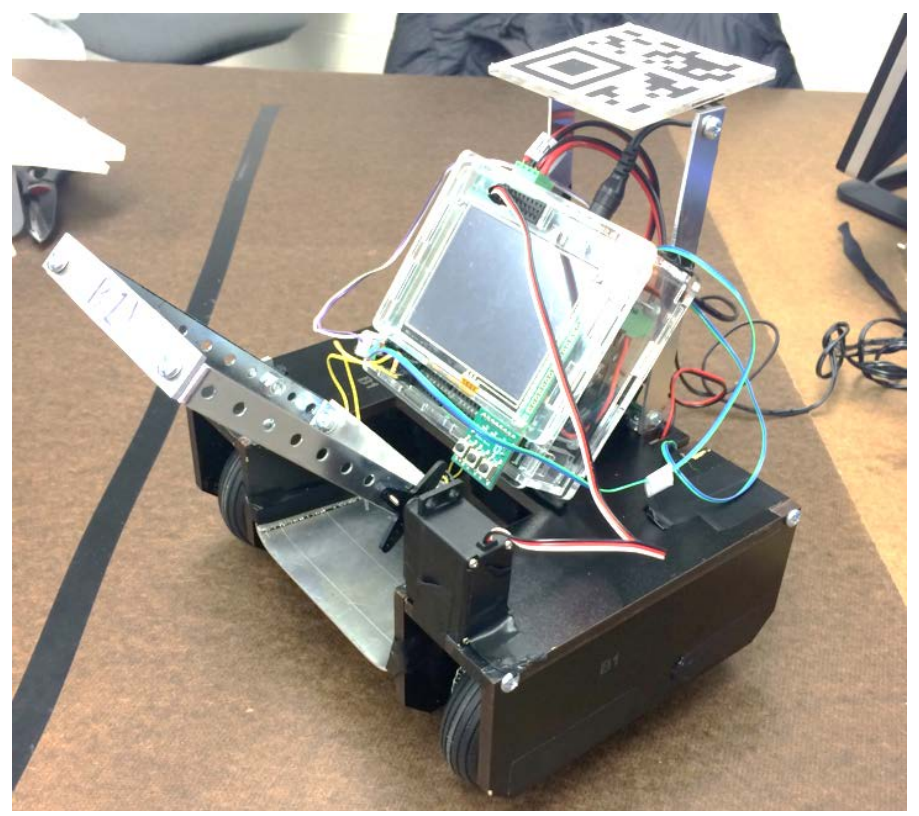

Figure 12: Fully Assembled Robot with Laser Cut, Laminated Wood Chassis 


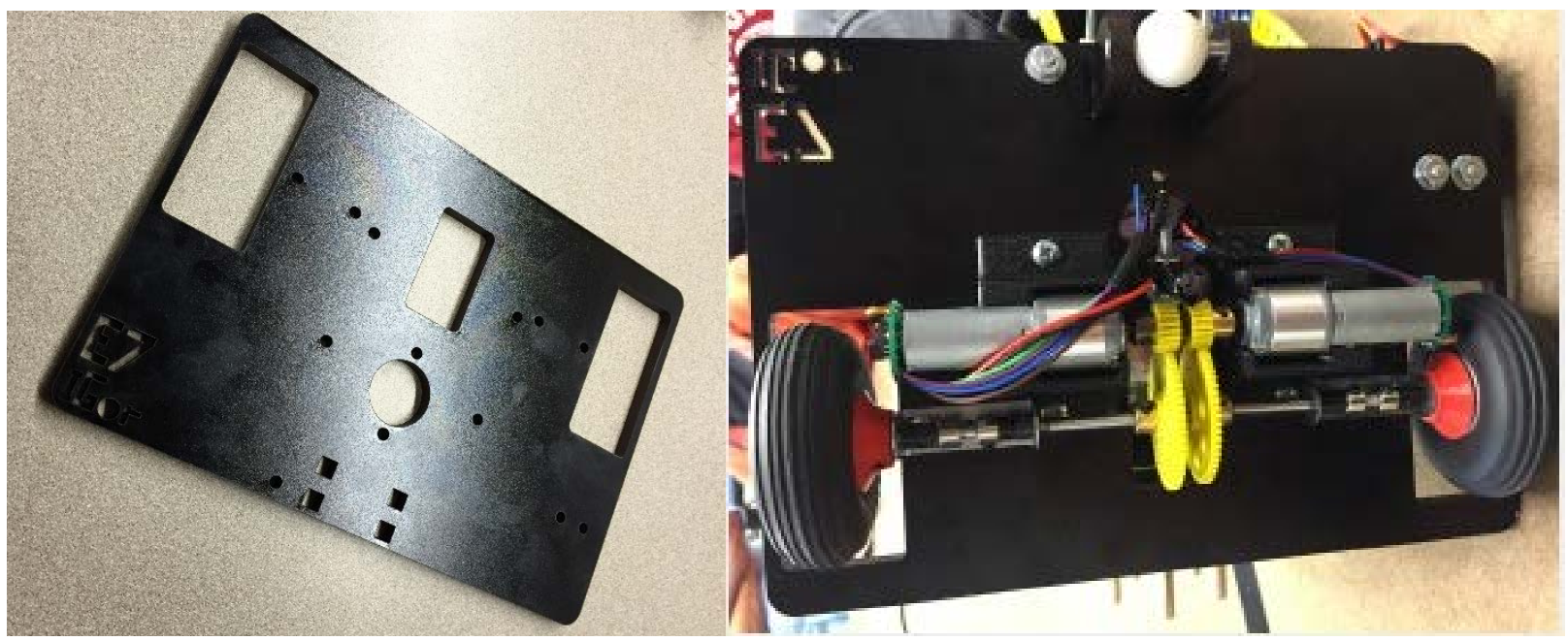

Figure 13: Laser Cut, Laminated Wood Chassis (Blank Chassis on Left, Drivetrain Assembly on Right)

\section{Custom Mechanism Design}

Other teams also choose to employ laser cutting to design mechanical parts that aid in completing course objectives. Figure 14 shows an image of a laser cut part used as a component of a mechanism to turn the Crank. The part has four holes for screws which line up with the holes of a servo motor attachment and an additional five holes to insert segments of dowel rods. The dowel rods line up in between the spokes of the Crank and the mechanism can be rotated with the servo motor.

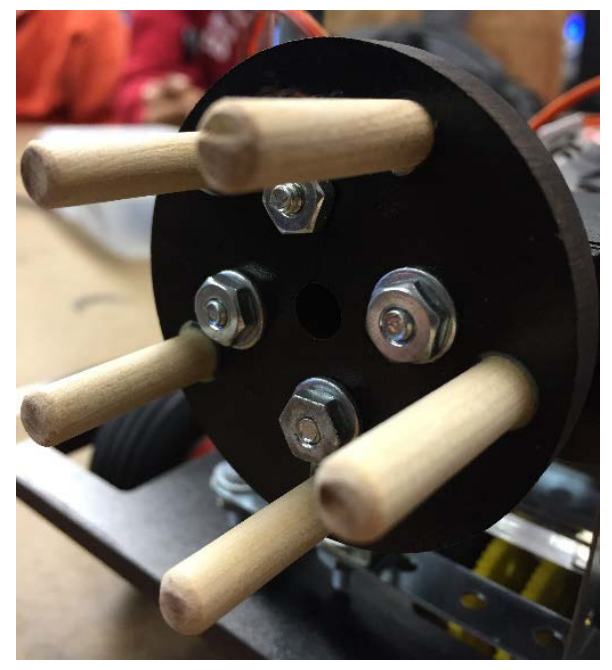

Figure 14: Laser Cut Servo Attachment used for turning the Crank

More complex mechanisms can be produced by using laser cutting as well. Figure 15 shown below depicts a robot that employs a large arm with three degrees of motion using three servo motors attached in different locations. The arm consists of seven laser cut pieces which serve as 
the structure of the arm and a U-shaped grabbing mechanism on the end. This multifunction arm can move up and down vertically, and the U-shaped mechanism can rotate in two directions much like a human wrist. This mechanism was used to complete multiple tasks due to its complexity and flexibility.

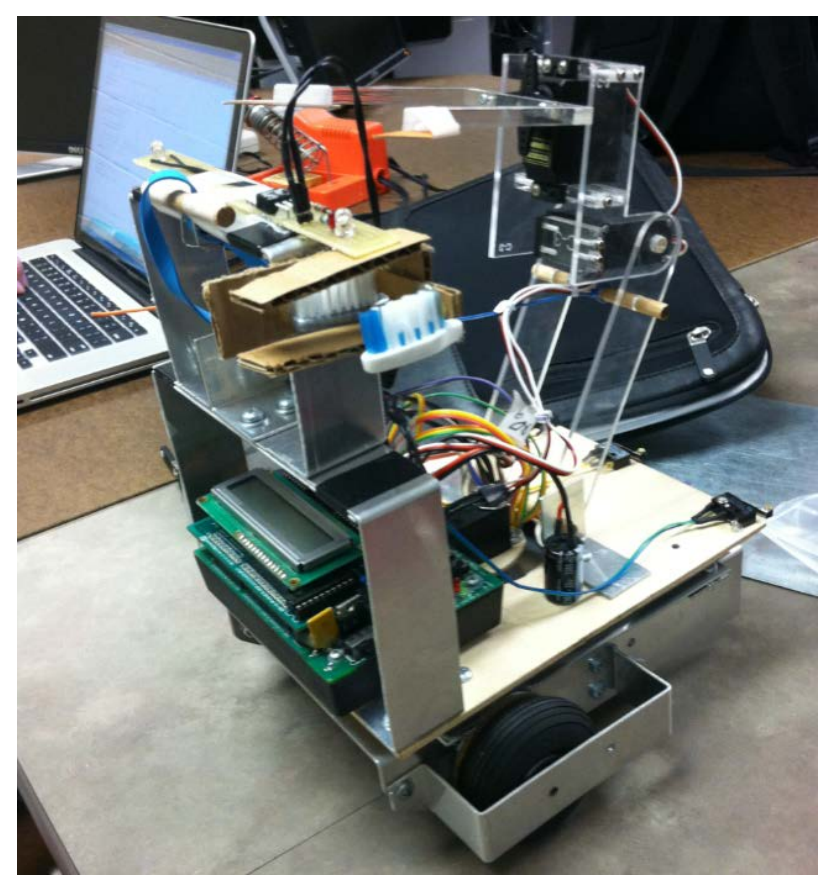

Figure 15: Complex 3-Servo Arm Mechanism made with Laser Cut Acrylic

\section{Discussion and Lessons Learned}

3D printing and laser cutting have proven to be extremely useful tools in this project. Each technology provides teams with the ability to increase the complexity and effectiveness of the robots they design and build. Although there is a significant time constraint on this project, the addition of rapid prototyping has led to better designed, well-engineered, more efficient, and more capable robots. These technologies have benefited the design project by not only aiding students in the ability to develop more complex ideas, but it has also allowed the program to develop more difficult course objectives. With this advanced technology and greater ability to design and fabricate innovative solutions to the objectives, FEH can create more intricate robot courses in the future without poorly impacting the student success rate in the project.

There are several important lessons learned and helpful hints listed below that will be useful for this project in the future.

- Many students come into the class with little knowledge of 3D printing or laser cutting, which can lead to structurally unstable parts. It may be beneficial in the future to 
introduce each technology further and expand on each by identifying good and bad design concepts of each.

- Students should test their laser cut chassis designs with some scrap material prior to submitting a file to the FEH Robot Store. Future modifications become extremely difficult once a piece has been laser cut.

- Students respond well to using these technologies and allowing the use of 3D printing and laser cutting actually aids in the process of learning to use more complicated features of Computer Aided Design software.

- Increased student engagement due to the addition of 3D printing and laser cutting has increased the level of complexity in robots resulting from this project.

\section{Summary and Conclusion}

This paper details the use of 3D printing and laser cutting technologies in a 10 week first-year engineering autonomous robot design project at The Ohio State University within the first-year Fundamentals of Engineering for Honors program and presents many innovative applications of each technology. Interdisciplinary four-person student teams are given a detailed Scenario document, describing all challenges and constraints of the project. Teams are challenged to design, build, and program a completely autonomous robot capable of performing a series of navigational and objective based tasks. 3D printing and laser cutting are offered to students to facilitate the design process, resulting in a plethora of custom, complex, and distinguishably different robots and mechanisms.

\section{References}

1. Freuler, R.J., M.J. Hoffmann, T.P. Pavlic, J.M. Beams, J.P. Radigan, P.K. Dutta, J.T. Demel, and E.D. Justen: "Experiences with a Comprehensive Freshman Hands-On Course - Designing, Building, and Testing Small Autonomous Robots", Proceedings of the 2003 American Society for Engineering Education Annual Conference, Nashville, Tennessee, June 2003. https://peer.asee.org/11882

2. Vernier, M.A., P.M. Wensing, C.E. Morin, A.H. Phillips, B.A. Rice, K.R. Wegman, C.P. Hartle, P.A. Clingan, K.M. Kecskemety, and R.J. Freuler: "Design of a Full-Featured Robot Controller for Use in a First-Year Robotics Design Project", Proceedings of the 2014 American Society for Engineering Education Annual Conference, Indianapolis, Indiana, June 2014. https://peer.asee.org/20260. Also published in the Computers in Education Division of ASEE Computers in Education Journal, Vol. XXV, No. 1, pp. 42-65, January-March, 2015. 